\title{
Correspondence between two antimatroid algorithmic characterizations
}

\author{
Yulia Kempner and Vadim E. Levit \\ Department of Computer Science \\ Holon Academic Institute of Technology \\ 52 Golomb Str., P.O. Box 305 \\ Holon 58102, ISRAEL \\ \{yuliak, levitv\}@hait.ac.il
}

Submitted: Aug 14, 2003; Accepted: Nov 6, 2003; Published: Nov 17, 2003

MR Subject Classifications: 90C27, 05B35

\begin{abstract}
The basic distinction between already known algorithmic characterizations of matroids and antimatroids is in the fact that for antimatroids the ordering of elements is of great importance.

While antimatroids can also be characterized as set systems, the question whether there is an algorithmic description of antimatroids in terms of sets and set functions was open for some period of time.

This article provides a selective look at classical material on algorithmic characterization of antimatroids, i.e., the ordered version, and a new unordered version. Moreover we empathize formally the correspondence between these two versions.
\end{abstract}

keywords: antimatroid, greedoid, chain algorithm, greedy algorithm, monotone linkage function.

\section{Introduction}

In this paper we compare two algorithmic characterization of antimatroids. There are many equivalent axiomatizations of antimatroids, that may be separated into two categories: antimatroids defined as set systems and antimatroids defined as languages. Boyd and Faigle [1] introduced an algorithmic characterization of antimatroids based on the language definition. Another characterization of antimatroids, that considers them as set systems, is the main topic of this paper. This characterization is based on the idea of optimization using set functions defined as minimum values of linkages between a set and the elements from the set complement. 
Section 2 gives some basic information about antimatroids as set systems and introduces truncated antimatroids. In Section 3 monotone linkage functions are considered. Optimization of the functions defined as minimums of monotone linkage functions extends to truncated antimatroids, and a polynomial algorithm finding an optimal set is constructed. In Section 4 the results of Boyd and Faigle are connected to our approach based on monotone linkage functions.

\section{Preliminaries}

Let $E$ be a finite set. A set system over $E$ is a pair $(E, \mathcal{F})$, where $\mathcal{F} \subseteq 2^{E}$ is a family of subsets of $E$, called feasible sets. We will use $X \cup x$ for $X \cup\{x\}$, and $X-x$ for $X-\{x\}$.

Definition 2.1 A non-empty set system $(E, \mathcal{F})$ is an antimatroid if

(A1) for each non-empty $X \in \mathcal{F}$, there is an $x \in X$ such that $X-x \in \mathcal{F}$

(A2) for all $X, Y \in \mathcal{F}$, and $X \nsubseteq Y$, there exist an $x \in X-Y$ such that $Y \cup x \in \mathcal{F}$.

Any set system satisfying $(A 1)$ is called accessible.

Definition 2.2 A set system $(E, \mathcal{F})$ has the interval property without upper bounds if for all $X, Y \in \mathcal{F}$ with $X \subseteq Y$ and for all $x \in E-Y, X \cup x \in \mathcal{F}$ implies $Y \cup x \in \mathcal{F}$.

There are some different antimatroid definitions:

Proposition 2.3 [2][3]For an accessible set system $(E, \mathcal{F})$ the following statements are equivalent:

(i) $(E, \mathcal{F})$ is an antimatroid

(ii) $\mathcal{F}$ is closed under union

(iii) $(E, \mathcal{F})$ satisfies the interval property without upper bounds.

For a set $X \in \mathcal{F}$, let $\Gamma(X)=\{x \in E-X: X \cup x \in \mathcal{F}\}$ be the set of feasible continuations of $X$. It is easy to see that an accessible set system $(E, \mathcal{F})$ satisfies the interval property without upper bounds if and only if for any $X, Y \in \mathcal{F}, X \subseteq Y$ implies $\Gamma(X) \cap(E-Y) \subseteq \Gamma(Y)$.

Definition 2.4 The $k$-truncation of a set system $(E, \mathcal{F})$ is a set system defined by

$$
\mathcal{F}_{k}=\{X \in \mathcal{F}:|X| \leq k\} \text {. }
$$

If $(E, \mathcal{F})$ is an antimatroid, then $\left(E, \mathcal{F}_{k}\right)$ is a $k$-truncated antimatroid [1].

The rank of a set $X \subseteq E$ is defined as $\varrho(X)=\max \{|Y|:(Y \in \mathcal{F}) \wedge(Y \subseteq X)\}$, the rank of the set system $(E, \mathcal{F})$ is defined as $\varrho(\mathcal{F})=\varrho(E)$. For a given antimatroid $(E, \mathcal{F})$ the rank of $k$-truncated antimatroid $\varrho\left(\mathcal{F}_{k}\right)=k$, whenever $k \leq \varrho(\mathcal{F})$. Notice, that every antimatroid $(E, \mathcal{F})$ is also a $k$-truncated antimatroid, where $k=\varrho(\mathcal{F})$. 
Clearly, a $k$-truncated antimatroid $(E, \mathcal{F})$ may not satisfy the interval property without upper bounds, but it does satisfy the following condition:

$$
\text { if } X, Y \in \mathcal{F}_{k-1} \text { and } X \subseteq Y \text {, then } x \in E-Y, X \cup x \in \mathcal{F} \text { imply } Y \cup x \in \mathcal{F} \text {. }
$$

A set system $(E, \mathcal{F})$ has the $k$-truncated interval property without upper bounds if it satisfies (1).

Theorem 2.5 An accessible set system $(E, \mathcal{F})$ of rank $k$ is a $k$-truncated antimatroid if and only if it satisfies the $k$-truncated interval property without upper bounds.

Proof. The only thing to show is that the set system $(E, \mathcal{F})$ with $k$-truncated interval property without upper bounds is a $k$-truncated antimatroid. To prove it one has to build an antimatroid generating the given set system by $k$-truncation. Define, by analogy with $[1]$

$$
\Omega=\left\{X \subseteq E \text { : there are some } X_{1}, \ldots, X_{p} \in \mathcal{F} \text { such that } X=X_{1} \cup \ldots \cup X_{p}\right\} .
$$

The set system $(E, \Omega)$ is closed under union. Hence to prove that $(E, \Omega)$ is an antimatroid we have only to verify that the set system $(E, \Omega)$ is accessible. Let $X \in \Omega$, and it has a decomposition $X=X_{1} \cup \ldots \cup X_{k}$. Then there exists $x \in X_{1}$ such that $X_{1}-x \in \mathcal{F}$. If $x \notin X_{2}, X_{3}, \ldots, X_{k}$, then $X-x=\left(X_{1}-x\right) \cup X_{2} \cup \ldots \cup X_{k} \in \Omega$, otherwise we could analyze the decomposition $X=\left(X_{1}-x\right) \cup X_{2} \cup \ldots \cup X_{k} \in \Omega$.

To show that the $k$-truncation of $(E, \Omega)$ is $(E, \mathcal{F})$ it is sufficient to prove that $X \in \mathcal{F}$ if and only if $X \in \Omega$ and $|X| \leq k$. Indeed, if $X \in \mathcal{F}$, then $|X| \leq k$, and $X \in \Omega$ by definition of $\Omega$. Conversely, let $X \in \Omega$ (i.e., there is a decomposition $X=A_{1} \cup \ldots \cup A_{p}$ ), and $|X| \leq k$. We show that $X \in \mathcal{F}$ by induction on $p$. If $p=1$, then, clearly, $X \in \mathcal{F}$. Consider $A=A_{1} \cup \ldots \cup A_{p-1}$. By the hypothesis of induction, $A \in \mathcal{F}$. Assume $|A|<k$, otherwise $X=A$ and then $X \in \mathcal{F}$. Since the set system $(E, \mathcal{F})$ is accessible, there exists a sequence of feasible sets $\emptyset=X_{0} \subset X_{1} \subset \ldots \subset X_{l}=A$ such that $X_{i}=X_{i-1} \cup x_{i}$ for $1 \leq i \leq l<k$. Assume $A \nsubseteq A_{p}$ and $\left|A_{p}\right|<k$, for if it is not true, then $X=A_{p}$, i.e., $X \in \mathcal{F}$. Let $j$ be the least integer for which $X_{j} \nsubseteq A_{p}$. Then $X_{j-1} \subseteq A_{p}, x_{j} \notin A_{p}$ and $X_{j-1} \cup x_{j} \in \mathcal{F}$, that together with (1) imply $A_{p} \cup x_{j} \in \mathcal{F}$. Going on with the increasing of the set $A_{p}$ we get the set $X=A_{p} \cup\left(A-A_{p}\right) \in \mathcal{F}$.

\section{The Chain Algorithm and monotone linkage func- tions}

In general, to optimize a set function is an NP-hard problem, but for some specific functions and for some specific set systems polynomial algorithms are known. In this section we investigate set functions defined as minimum values of monotone linkage functions. Such set functions can be maximized by a greedy type algorithm over a family of all subsets of $E$ (see [7]). Here we extend this result to antimatroids. 
Monotone linkage functions were introduced by Mullat [6]. We will give some necessary basic notions.

Let $\pi: E \times 2^{E} \rightarrow \mathbf{R}$ be a monotone linkage function such that

$$
\text { if } X, Y \subseteq E \text { and } x \in E \text {, then } X \subseteq Y \text { implies } \pi(x, X) \geq \pi(x, Y) \text {. }
$$

For example, $\pi(x, X)=\min _{y \in X} d_{x y}$, where $d_{x y}$ is a distance between two objects, is a monotone linkage function.

Consider $F: 2^{E} \rightarrow \mathbf{R}$ defined for each $X \subset E$

$$
F(X)=\min _{x \in E-X} \pi(x, X) .
$$

These functions were studied in [7],[4], where a simple polynomial algorithm finding a set $X \subset E$ such that

$$
F(X)=\max \{F(Y): Y \subset E\}
$$

was developed. The idea of this algorithm was also used in searching of a protein sequence alignment [5]. In this section we extend these results to truncated antimatroids. For this purpose we define on a set system $(E, \mathcal{F})$ a new set function as follows:

$$
F_{\mathcal{F}}(X)=\left\{\begin{array}{ll}
\min _{x \in \Gamma(X)} \pi(x, X), & \Gamma(X) \neq \emptyset \\
-\infty, & \text { otherwise }
\end{array} .\right.
$$

It should be pointed out that the definition (5) is not limited to antimatroids, but for each $k$-truncated antimatroid $(E, \mathcal{F})$, the function $F_{\mathcal{F}}$ is well defined $(\neq-\infty)$ on the set system $\left(E, \mathcal{F}_{k-1}\right)$.

Consider the following optimization problem.

Given: a monotone linkage function $\pi$, and a set $\operatorname{system}(E, \mathcal{F})$.

Find: the feasible set $X \in \mathcal{F}$ such that $F_{\mathcal{F}}(X)=\max \left\{F_{\mathcal{F}}(Y): Y \in \mathcal{F}\right\}$, where function $F_{\mathcal{F}}$ defined by $(5)$.

To solve this problem we build the following algorithm.

The Chain Algorithm $(E, \mathcal{F}, \pi)$

1. Set $X^{0}:=\emptyset$

2. Set $X:=\emptyset$

3. While $\Gamma(X) \neq \emptyset$ do

3.1 If $F_{\mathcal{F}}(X)>F_{\mathcal{F}}\left(X^{0}\right)$, set $X^{0}:=X$

3.2 Choose $x \in \Gamma(X)$ such that $\pi(x, X) \leq \pi(y, X)$ for all $y \in \Gamma(X)$

3.3 Set $X:=X \cup x$

4. Return $X^{0}$

Thus, the Chain Algorithm generates the chain of sets

$$
\emptyset=X_{0} \subset X_{1} \subset \ldots \subset X_{k},
$$

where $X_{i}=X_{i-1} \cup x_{i}$ and $x_{i} \in \Gamma\left(X_{i-1}\right)$ for $1 \leq i \leq k$, and returns the minimal set $X^{0}$ of the chain on which the value $F_{\mathcal{F}}\left(X^{0}\right)$ is maximal. 
Theorem 3.1 Let $(E, \mathcal{F})$ be an accessible set system of rank $k$. If the set of feasible continuations of $X$ is not empty for each $X \in \mathcal{F}_{k-1}$, then the following statements are equivalent:

(1) the set system $(E, \mathcal{F})$ is a $k$-truncated antimatroid.

(2) The Chain Algorithm finds a feasible set that maximizes the function $F_{\mathcal{F}}$ for every monotone linkage function $\pi$.

Proof. Let $X^{0}$ be the set obtained by the Chain Algorithm. To prove that $X^{0}$ is a feasible set maximizing $F_{\mathcal{F}}$, we have to show that $F_{\mathcal{F}}(X) \leq F_{\mathcal{F}}\left(X^{0}\right)$ for each $X \in \mathcal{F}_{k-1}$. Let $X_{0} \subset X_{1} \subset \ldots \subset X_{k}$ be the chain generated by the Chain Algorithm. Let $j$ be the least integer for which $X_{j} \nsubseteq X$. Then $X_{j-1} \subseteq X, x_{j} \notin X$ and $X_{j-1} \cup x_{j} \in \mathcal{F}$, that implies (from (1)) $x_{j} \in \Gamma(X)$. Hence,

$$
F_{\mathcal{F}}(X) \leq \pi\left(x_{j}, X\right) \leq \pi\left(x_{j}, X_{j-1}\right)=F_{\mathcal{F}}\left(X_{j-1}\right) \leq F_{\mathcal{F}}\left(X^{0}\right) .
$$

Conversely, consider an accessible set system $(E, \mathcal{F})$ that is not $k$-truncated antimatroid, i.e., there exists $A, B \in \mathcal{F}_{k-1}$ such that $A \subset B$, and there is $a \in E-B$ such that $A \cup a \in \mathcal{F}$ and $B \cup a \notin \mathcal{F}$. Accessibility of the set system $(E, \mathcal{F})$ implies that there exists a sequence of feasible sets

$$
\emptyset=A_{0} \subset A_{1} \subset \ldots \subset A_{p}=A \subset A_{p+1}=A \cup a,
$$

where $A_{i}=A_{i-1} \cup a_{i}$ for $1 \leq i \leq p$, and $a_{p+1}=a$. Define a monotone linkage function $\pi$ on pairs $(x, X)$ where $X \subset E$ and $x \in E-X$ :

$$
\pi(x, X)=\left\{\begin{array}{ll}
1, & X \supseteq A_{i-1} \text { and } x=a_{i} \text { or } A \cup a \subseteq X \subset E \text { and } x \in E-X \\
2, & \text { otherwise. }
\end{array} .\right.
$$

Then the Chain Algorithm generates a chain $A_{0} \subset \ldots \subset A_{p} \subset A_{p+1} \subset \ldots \subset A_{k}$, on which the values of the function $F_{\mathcal{F}}$ are equal to 1 , but $F_{\mathcal{F}}(B)=2$. Thus, the Chain Algorithm does not find a feasible set that maximizes the function $F_{\mathcal{F}}$.

The Chain Algorithm is a greedy type algorithm since it is based on the best choice principle: it chooses on each step the extreme element (with respect to the linkage function) and, thus, approaches the optimal solution. Let $P$ is the maximum complexity of $\pi(x, X)$ computation over all pairs $(x, X)$, where $x \in E-X$. Then the Chain Algorithm finds the optimal feasible set in $O\left(P|E|^{2}\right)$ time. For example, in some clustering problems the complexity of the Chain Algorithm is $O\left(|E|^{3}\right.$ ) (see [4]).

\section{Correspondence between two algorithmic charac- terization of antimatroids}

In this section we consider an algorithmic approach to antimatroids due to Boyd and Faigle [1]. Their idea is based on the definition of an antimatroid as a formal language. 
Given a finite alphabet $E$ consists of letters. A word over $E$ is a sequence of letters from $E$, denoted by the lower case of Greek letters $\alpha, \beta$ and $\gamma$. A language $\mathcal{L}$ is a set of words of $E$. The concatenation of two words $\alpha$ and $\beta$ will be denoted $\alpha \beta, \alpha_{k}$ will be used to denote a word of length $k$ and the set of distinct letters in a word $\alpha$ will be denoted $\widetilde{\alpha}$. The language is called simple if there are no words with repeated letters.

Definition 4.1 An antimatroid language is a simple language $(E, \mathcal{L})$ satisfying the following two properties:

(1) If $\alpha x \in \mathcal{L}$, then $\alpha \in \mathcal{L}$.

(2) If $\alpha, \beta \in \mathcal{L}$ and $\widetilde{\alpha} \nsubseteq \widetilde{\beta}$, then there exists an $x \in \widetilde{\alpha}$ such that $\beta x \in \mathcal{L}$.

Antimatroids and antimatroid languages are equivalent in the following sense [3].

Theorem 4.2 If $(E, \mathcal{L})$ is an antimatroid language, then

$$
F(\mathcal{L})=\{\widetilde{\alpha}: \alpha \in \mathcal{L}\}
$$

is an antimatroid $(E, F(\mathcal{L}))$.

Conversely, if $(E, \mathcal{F})$ is an antimatroid, then

$$
L(\mathcal{F})=\left\{x_{1} \ldots x_{k}:\left\{x_{1}, \ldots x_{j}\right\} \in \mathcal{F} \text { for } 1 \leq j \leq k\right\}
$$

is an antimatroid language $(E, L(\mathcal{F}))$. Further, $L(F(\mathcal{L}))=\mathcal{L}$ and $F(L(\mathcal{F}))=\mathcal{F}$.

The next problem is considered in [1]: let $f: E \times 2^{E} \rightarrow \mathbf{R}$ be a monotone function such that $f(x, A) \leq f(x, B)$ whenever $B \subseteq A$. Define a maximum nesting function

$$
W\left(x_{1} \ldots x_{k}\right)=\max \left\{f\left(x_{1},\left\{x_{1}\right\}\right), \ldots, f\left(x_{k},\left\{x_{1}, \ldots, x_{k}\right\}\right)\right\} .
$$

The minimax nesting problem is defined as follows: given a simple language $(E, \mathcal{L})$ with a monotone function $f$ and a nonnegative integer $k \leq \varrho(\mathcal{L})$, find $\alpha_{k} \in \mathcal{L}$ such that

$$
W\left(\alpha_{k}\right)=\min \left\{W\left(\beta_{k}\right): \beta_{k} \in \mathcal{L}\right\} .
$$

The main theorem proved in [1] reads as follows.

Theorem 4.3 Let $(E, \mathcal{L})$ be a simple language. The greedy algorithm solves the minimax nesting problem for every monotone function $f$ if and only if $(E, \mathcal{L})$ is a truncated antimatroid.

In the sequel we will discuss the correspondence between the set system and language characterizations of antimatroids.

Firstly, the word $\alpha_{k}=x_{1} \ldots x_{k}$ constructed with the greedy algorithm satisfies also the following property:

$$
W\left(x_{1} \ldots x_{i}\right)=\min \left\{W\left(\beta_{i}\right): \beta_{i} \in \mathcal{L}\right\} \text { for each } i \text { such that } 1 \leq i \leq k
$$

(see [1]).

Secondly, the Chain Algorithm builds a sequence $\emptyset=X_{0} \subset X_{1} \subset \ldots \subset X_{k}$, where $X_{i}=X_{i-1} \cup x_{i}$ for $1 \leq i \leq k$, i.e., the algorithm generates the sequence $x_{1} \ldots x_{k}$. So every set $X_{i}$, obtained by the Chain Algorithm, has a natural order: $X_{i}=\left\{x_{1}, \ldots, x_{i}\right\}$, i.e., we can interpret each set $X_{i}$ as a word $\alpha_{i}=x_{1} \ldots x_{i}$. Now we are ready to prove the following. 
Theorem 4.4 Let $(E, \mathcal{L})$ be a $k$-truncated antimatroid and let

$$
f\left(x_{i},\left\{x_{1}, \ldots, x_{i}\right\}\right)=\pi\left(x_{i},\left\{x_{1}, \ldots, x_{i-1}\right\}\right) \text { for each } i \text { such that } 1 \leq i \leq k
$$

then

(i) if $X^{0}$ is an optimal set obtained by the Chain Algorithm, then there exists a word $\alpha_{k} \in \mathcal{L}$ that satisfies (6) and $X^{0}=\left\{x_{1}, \ldots, x_{p}\right\}$ is a shortest prefix of $\alpha_{k}$ such that $W\left(x_{1} \ldots x_{p+1}\right)=W\left(\alpha_{k}\right)=F_{\mathcal{L}}\left(X^{0}\right)$.

(ii) if $\alpha_{k}$ is a solution of the minimax nesting problem obtained by the greedy algorithm, then a shortest prefix $\left\{x_{1}, \ldots, x_{p}\right\}$ of $\alpha_{k}$ such that $W\left(x_{1} \ldots x_{p+1}\right)=W\left(\alpha_{k}\right)$ maximizes the function $F_{\mathcal{L}}$.

Proof. $(i)$ Let $x_{1} \ldots x_{k}$ be the sequence generating by the Chain Algorithm and let $X^{0}=$ $\left\{x_{1}, \ldots, x_{p}\right\}$. Set $\alpha_{k}=x_{1} \ldots x_{k}$ and prove that $\alpha_{k}$ satisfies (6). Suppose that the opposite is true, then let $\gamma_{m}=y_{1} \ldots y_{m}$ be a shortest word such that $W\left(\gamma_{m}\right)<W\left(x_{1} \ldots x_{m}\right)$. It means that for each $i<m$

$$
\max \left\{\pi\left(x_{1}, \emptyset\right), \ldots, \pi\left(x_{i},\left\{x_{1}, \ldots, x_{i-1}\right\}\right)\right\} \leq \max \left\{\pi\left(y_{1}, \emptyset\right), \ldots, \pi\left(y_{i},\left\{y_{1}, \ldots, y_{i-1}\right\}\right)\right\}
$$

and for each $i \leq m$

$$
\pi\left(x_{m},\left\{x_{1}, \ldots, x_{m-1}\right\}\right)>\max \left\{\pi\left(y_{1}, \emptyset\right), \ldots, \pi\left(y_{i},\left\{y_{1}, \ldots, y_{i-1}\right\}\right)\right\} .
$$

If $\left\{y_{1} \ldots y_{m-1}\right\}=\left\{x_{1}, \ldots, x_{m-1}\right\}$, then $y_{m} \in \Gamma\left(\left\{x_{1}, \ldots, x_{m-1}\right\}\right)$, and by $(7)$

$$
\pi\left(y_{m},\left\{x_{1}, \ldots, x_{m-1}\right\}\right)=\pi\left(y_{m},\left\{y_{1}, \ldots, y_{m-1}\right\}\right)<\pi\left(x_{m},\left\{x_{1}, \ldots, x_{m-1}\right\}\right) .
$$

So the Chain Algorithm should choose $y_{m}$ and not $x_{m}$.

Thus, let $j$ be the smallest index such that $\left\{y_{1}, \ldots, y_{j-1}\right\} \subseteq\left\{x_{1}, \ldots, x_{m-1}\right\}$ and $y_{j} \notin$ $\left\{x_{1}, \ldots, x_{m-1}\right\}$. Since $y_{j} \in \Gamma\left(\left\{y_{1}, \ldots, y_{j-1}\right\}\right)$, by $k$-truncated interval property without upper bounds we get that $y_{j} \in \Gamma\left(\left\{x_{1}, \ldots, x_{m-1}\right\}\right)$. Hence, monotonicity of $\pi$ and (7) imply

$$
\pi\left(y_{j},\left\{x_{1}, \ldots, x_{m-1}\right\}\right) \leq \pi\left(y_{j},\left\{y_{1}, \ldots, y_{j-1}\right\}\right)<\pi\left(x_{m},\left\{x_{1}, \ldots, x_{m-1}\right\}\right)
$$

which contradicts the optimal choice of $x_{m}$.

Finally, the Chain Algorithm builds $X^{0}=\left\{x_{1}, \ldots, x_{p}\right\}$, which is the shortest prefix of $\alpha_{k}$ such that

$$
F_{\mathcal{L}}\left(X^{0}\right)=\pi\left(x_{p+1},\left\{x_{1} \ldots x_{p}\right\}\right)=W\left(x_{1} \ldots x_{p+1}\right)=W\left(\alpha_{k}\right) .
$$

(ii) Conversely, let $\alpha_{k}$ be a solution of the minimax nesting problem and let $X^{0}=$ $x_{1}, \ldots, x_{p}$ be the shortest prefix such that $W\left(x_{1} \ldots x_{p+1}\right)=W\left(\alpha_{k}\right)$. Then

$$
\pi\left(x_{p+1},\left\{x_{1} \ldots x_{p}\right\}\right)>\pi\left(x_{i+1},\left\{x_{1} \ldots x_{i}\right\}\right) \text { for } i<p,
$$

and

$$
\pi\left(x_{p+1},\left\{x_{1} \ldots x_{p}\right\}\right) \geq \pi\left(x_{i+1},\left\{x_{1} \ldots x_{i}\right\}\right) \text { for } i \geq p \text {. }
$$


Certainly, $\pi\left(x_{p+1},\left\{x_{1} \ldots x_{p}\right\}\right)=\min _{x \in \Gamma\left(X^{0}\right)} \pi\left(x,\left\{x_{1} \ldots x_{p}\right\}\right)$. If not, there is $x^{0} \in \Gamma\left(X^{0}\right)$ such that $\pi\left(x^{0},\left\{x_{1} \ldots x_{p}\right\}\right)<\pi\left(x_{p+1},\left\{x_{1} \ldots x_{p}\right\}\right)$, i.e. $W\left(x_{1} \ldots x_{p} x^{0}\right)<W\left(x_{1} \ldots x_{p+1}\right)$ - contradiction with (6). So, $F_{\mathcal{L}}\left(X^{0}\right)=\pi\left(x_{p+1},\left\{x_{1} \ldots x_{p}\right\}\right)$.

Consider some set $X \in F(\mathcal{L})$. If $X=\left\{x_{1} \ldots x_{j}\right\}$ (i.e., $X$ is a prefix of $\alpha_{k}$ ), then

$$
F_{\mathcal{L}}(X)=\min _{x \in \Gamma(X)} \pi(x, X) \leq \pi\left(x_{j+1},\left\{x_{1} \ldots x_{j}\right\}\right) \leq \pi\left(x_{p+1},\left\{x_{1} \ldots x_{p}\right\}\right)=F_{\mathcal{L}}\left(X^{0}\right) .
$$

Otherwise, let $j$ be the smallest index such that $\left\{x_{1} \ldots x_{j}\right\} \subseteq X$ and $x_{j+1} \notin X$. Then $x_{j+1} \in \Gamma(X)$ by 1 . Hence,

$$
\begin{gathered}
F_{\mathcal{L}}(X)=\min _{x \in \Gamma(X)} \pi(x, X) \leq \pi\left(x_{j+1}, X\right) \leq \\
\leq \pi\left(x_{j+1},\left\{x_{1} \ldots x_{j}\right\}\right) \leq \pi\left(x_{p+1},\left\{x_{1} \ldots x_{p}\right\}\right)=F_{\mathcal{L}}\left(X^{0}\right) .
\end{gathered}
$$

\section{Conclusions}

In this article, we discussed a set system algorithmic description of one subclass of greedoids, namely, antimatroids. Further we compared a new description with a known one based on the approach defining greedoids as languages. Actually, there are some more important subclasses of greedoids also enjoying natural algorithmic characterizations in terms of their feasible set systems, for instance, matroids and Gaussian greedoids. These findings may lead to new algorithmic frameworks for additional types of greedoids. We consider the family of interval greedoids as a strong candidate for the collection of successes of the set system algorithmic approach.

\section{References}

[1] E.A. Boyd, and U. Faigle, An algorithmic characterization of antimatroids, Discrete Applied Mathematics 28 (1990) 197-205

[2] A.Björner and G.M.Ziegler, Introduction to greedoids, in "Matroid applications", ed. N. White, Cambridge University Press, Cambridge, UK,1992

[3] B.Korte, L.Lovász, and R.Schrader, Greedoids, Springer-Verlag, New York/Berlin, 1991

[4] Y.Kempner, B.Mirkin, and I.Muchnik, Monotone linkage clustering and quasi-concave functions, Appl.Math.Lett. 10 ,No.4 (1997) 19-24

[5] C.Kulikowski, I.Muchnik and L.Shvartser, Multiple sequence alignment using the quasi-concave function optimization based on the DIALIGN combinatorial structures, DIMACS Technical Report 2001-02 (2001) 
[6] J.Mullat, Extremal subsystems of monotone systems: I, II, Automation and Remote Control 37, (1976) 758-766; 1286-1294

[7] Y.Zaks (Kempner), and I.Muchnik, Incomplete classifications of a finite set of objects using monotone systems, Automation and Remote Control 50, (1989), 553-560 